\title{
Interacciones sociales, prácticas rituales y procesos de cambio durante los periodos Tardío e Inca (ca. 900- 1536 d.C.) en la cuenca de Ratones, Puna de Salta
}

\section{(1) Federico I. Coloca"}

Fecha de defensa: 13 de noviembre de 2017

Director: Dr. Gabriel E. J. López

Co-director: Dr. Hernán J. Muscio

Jurados: Dres. Daniel Olivera, José M. Vaquer

y Verónica Williams

\section{Introducción a la problemática}

El tema de investigación de la tesis fue el estudio de la diversidad arqueológica de las ocupaciones humanas en la cuenca del salar de Ratones, Puna de Salta, Noroeste argentino, durante los períodos Tardío e Inca (ca. 9001536 d.C.). Específicamente, se integró la información arqueológica recuperada en dos sitios denominados Abra de Minas y Cueva Inca Viejo. Ambos sitios están siendo estudiados de forma integrada ya que presentan información complementaria. En este contexto, la tesis se estructuró en base a tres temas importantes para la comprensión de las ocupaciones humanas en la región:

1) Los procesos de interacción macrorregional, considerando el uso de la cuenca como un espacio estratégico en la circulación de bienes y recursos y principalmente en la transmisión cultural entre individuos y/o grupos;

2) La caracterización de espacios sacralizados o rituales, teniendo en cuenta la importancia de la simbología y las prácticas rituales como formas de transmisión de información para la cohesión y diferenciación de grupos y para la legitimación y el control social;

3) El impacto de la ocupación inca en el área, evaluando los procesos de cambio ocurridos y las estrategias de dominación implementadas por el Imperio, las cuales incluyen un punto de vista político, relacionado con el control del uso del espacio; económico, asociado con la explotación minera del área; y también simbólico, a partir de la propiciación de rituales y materialidades relacionadas con prácticas ceremoniales.

\section{Área de estudio}

La cuenca del salar de Ratones conforma un área que comenzó a ser investigada recientemente (Coloca,

* Instituto de Arqueología, Facultad de Filosofía y Letras, Universidad de Buenos Aires (UBA) - CONICET, 25 de Mayo 217, $3^{\circ}$ piso (CP C1002ABE) Ciudad Autónoma de Buenos Aires, Argentina. E-mail: fedeigco@hotmail.com
2017; López y Coloca, 2015; López, Coloca y Orsi, 2009; López, Coloca, Araya, Orsi y Seguí, 2015). La misma, por encontrarse localizada entre la Puna Norte y Sur y cercana a los valles mesotermales (aproximadamente entre 60 y 100 $\mathrm{km}$ ), podría haber sido un lugar de paso relevante dentro de una ruta caravanera para el traslado de bienes, recursos y, especialmente, de comunicación entre individuos y/o grupos. En el período Tardío esta cuenca se habría relacionado con el sector central del valle Calchaquí, en donde se han registrado evidencias de ocupaciones con cronologías desde el Formativo hasta épocas históricas (Baldini, Baffi, Quiroga y Villamayor, 2004; Cremonte y Williams, 2007). De esta manera, es posible suponer que la cuenca de Ratones estuviera articulada con dichas ocupaciones del valle medio Calchaquí y que bajo el dominio incaico se haya conformado como un nodo dentro de una red de instalaciones estatales.

En cuanto a los sitios estudiados, Abra de Minas es un asentamiento a cielo abierto ubicado a $4.250 \mathrm{~m}$ s.n.m. El mismo se destaca por registrar más de 90 estructuras, en su mayoría de planta rectangular y cuadrangular y una alta frecuencia de material arqueológico en superficie (López y Coloca, 2015). La evidencia arquitectónica y artefactual de este sitio indica una intensa ocupación incaica. Por su parte, Cueva Inca Viejo se encuentra a $2 \mathrm{~km}$ de Abra de Minas, a una altura de $4.312 \mathrm{~m}$ s.n.m y se caracteriza por la presencia de arte rupestre y materiales procedentes de distintas regiones, en particular de tierras más bajas como las yungas, ubicadas a casi 300 km (López et al., 2015).

\section{Marco Teórico}

Se planteó un marco teórico que sirviera como base para comprender y discutir las diferentes problemáticas sobre las ocupaciones tardías e incas de la cuenca de Ratones. Para ello, desde una perspectiva evolutiva se consideraron las propuestas desarrolladas por la hipótesis de los compromisos grupales y la teoría de la transmisión cultural (Richerson y Boyd, 2000).

La hipótesis de los compromisos grupales sostiene que los humanos se encuentran adaptados a vivir en grupos. Esta predisposición se encuentra determinada por emociones y mecanismos cognitivos que desarrollan cohesión grupal, conteniendo compromisos que se encuentran arraigados profundamente en los individuos. A partir de esta perspectiva evolutiva, se postula que las normas, 
las instituciones y la acción colectiva que delimitan a las sociedades contienen rasgos que remiten o responden a la propensión de los individuos a comprometerse a un grupo y se relacionan con los sentimientos más básicos (parentesco y reciprocidad) (Richerson y Boyd, 2000; Richerson y Henrich, 2009).

Por otra parte, la teoría de la transmisión cultural permite comprender los mecanismos de reproducción y herencia de valores culturales, la cooperación y coordinación al interior de un grupo y la diferenciación entre grupos (delimitador). Este marco es adecuado, a su vez, para la comprensión de sistemas rituales. Los rituales se constituyen como prácticas vinculadas con la transmisión de marcadores culturales simbólicos, tanto para la cohesión de grupo como para la diferenciación o demarcación de límites con otros grupos (Richerson y Henrich, 2009).

Esta perspectiva teórica permite discutir los procesos de interacción social de escala amplia que se desarrollaron en contextos prehispánicos tardíos e incaicos y el rol de los espacios rituales para reforzar los compromisos de los individuos hacia sus grupos de pertenencia, como también dentro de las estrategias de cohesión de grupos y dominación por parte del imperio. Se debe considerar que, en diversos contextos, los incas usaron las prácticas rituales como forma de legitimación de su control y/o propiedad sobre territorios y sistemas productivos. Este es el caso de la relación entre los sitios mineros y los rituales de producción en el norte de Chile (Salazar, Berenguer y Vega, 2013; Soto Rodríguez y Salazar, 2016). En contextos rurales andinos la dimensión económica tiene su contraparte en la dimensión simbólica expresada en rituales de producción (Martel, 2011; Van Kessel, 1989). Esto se ha denominado en otros contextos estatales como apropiación simbólica de espacios productivos (Williams, Villegas, Gheggi y Chaparro, 2005). Dichos rituales pueden haber cumplido distintos roles en estas sociedades. Concretamente, se trata de prácticas vinculadas con la transmisión de información, ya sea para la cohesión de los grupos y/o para la legitimación y el control, entre otras posibilidades.

\section{Procedimientos Metodológicos}

El estudio del registro arqueológico se llevó adelante mediante una metodología distribucional. Para ello se realizaron distintas tareas. Por un lado, se plantearon prospecciones y transectas sistemáticas con recolección de material superficial. A su vez, se llevó a cabo el relevamiento del área considerando diferentes indicadores de un uso más intenso del espacio. Finalmente, se realizaron sondeos y cuadrículas en distintas partes de los sitios, con el objetivo de efectuar un reconocimiento estratigráfico y cronológico.

La tesis se apoyó en el análisis de la evidencia arquitectónica y cerámica de los sitios estudiados, ya que se consideran los indicadores diagnósticos más relevantes y que envuelven información en amplias escalas espacio-temporales. A su vez, también fueron estudiadas otras líneas de análisis que aportaron valiosa información (lítico, arte rupestre, macrorrestos vegetales, entre otros).

En cuanto al marco cronológico, se consideró un bloque temporal que envuelve diferentes procesos y que en líneas generales comienza ca. 900 d.C. y culmina ca. 1536 d.C. Dentro de dicho lapso y basándonos en la bibliografía referida al tema (Nielsen, 2003; Pérez Gollán, 1973; Raffino, 1981; Tarragó, 2000; entre otros), se denominaron el período Tardío (designado también como Desarrollos Regionales) como un rango cronológico que abarca entre ca. 900 y 1430 d.C. y el período Inca entre ca. 1430 y 1536 d.C. La cronología del área se conformó a partir de fechados radiocarbónicos. Asimismo, se consideraron diferentes artefactos arqueológicos diagnósticos que permitieron complementar los datos de carbono 14.

\section{Discusión de los resultados}

Los resultados arqueológicos alcanzados permitieron discutir los objetivos planteados en base a los tres ejes o temas de importancia que guiaron la tesis. En primer lugar, el conjunto de evidencia sugiere que las prácticas rituales en la cuenca de Ratones pudieron haber funcionado para estimular el compromiso de los individuos hacia sus grupos, como también para marcar diferencias entre ellos. Dado que los primeros y más fuertes compromisos son aquellos relacionados con la familia y el parentesco, la replicación de rituales que apelan a estas emociones, tales como el culto a los ancestros, permite transmitir información cultural para cohesionar los grupos más allá de la cercanía parental. En el período Tardío, distintas formas de transmisión cultural habrían permitido compartir y fijar comportamientos de cohesión en los grupos pastoriles-caravaneros como así también señalar diferencias en relación con otros grupos. En este marco el arte rupestre habría funcionado como marcador simbólico. Durante la ocupación inca, los indicadores arqueológicos evidenciados permiten sostener la hipótesis de legitimación y control económico-ritual por parte de los incas. Esto habría incluido ceremonias de hospitalidad y una apelación a los compromisos grupales previos de las poblaciones locales, a través del control del culto a los ancestros y de los rituales de producción.

Por otra parte, la evidencia recuperada en la cuenca de Ratones aporta información relevante sobre el rol que pudo jugar el área dentro del tráfico macrorregional para los períodos Tardío e Inca. Dentro de este contexto, el tráfico caravanero desempeñó un rol importante, mediante la circulación de materiales pertenecientes a diferentes regiones como también en la reproducción de ideas y traspaso de información mediante el arte rupestre. 
Con la ocupación incaica del área, aumentó la escala y la intensidad del tráfico. En este marco, la circulación de cerámica estatal, como la de otros artefactos valiosos para el imperio, pudo jugar un rol de importancia en la transmisión de la ideología inca.

Finalmente, los estudios realizados hasta el momento permiten discutir tanto los aspectos políticos y económicos como los simbólicos desarrollados por el imperio incaico en la cuenca de Ratones. En primer lugar, los incas impusieron un cambio en el área en base a una fuerte presencia política por medio de la construcción de arquitectura estatal de forma estratégica. Por otra parte, desde el punto de vista económico, el cambio que produjo la irrupción imperial sobre el área se ve reflejado en la imposición de un nuevo espacio vinculado con la extracción de minerales. En este sentido, la evidencia de Cueva Inca Viejo y la alta inversión de trabajo en Abra de Minas a más 4.200 m s.n.m, sugieren que la explotación minera de turquesa pudo ser una de las causas principales de la ocupación inca del área. Por último, la apropiación simbólica por medio del control de lugares sagrados así como la propiciación de festividades de hospitalidad fue parte de una estrategia de dominación efectiva que el Estado incaico implementó en Ratones.

\section{Referencias citadas}

》 Baldini, L., Baffi, E., Quiroga, L. y Villamayor, V. (2004). Los desarrollos regionales en el valle Calchaquí Central, Salta. Relaciones de la Sociedad Argentina de Antropología, XXIX, 59-80.

" Coloca, F. I. (2017). La arquitectura de Abra de Minas, un sitio con evidencias tardías/incaicas en la puna de Salta, Argentina. Intersecciones en Antropología, 18(2), 245-256.

"Cremonte, B. y Williams, V. (2007). La construcción social del paisaje durante la dominación inka en el noroeste argentino. En A. E. Nielsen, M. C. Rivolta, V. Seldes, M. M. Vázquez y P. H. Mercolli (Comps.), Procesos sociales prehispánicos en el sur andino. La vivienda, la comunidad y el territorio (pp. 207-236). Córdoba: Brujas.

» López, G. y Coloca, F. (2015). El sitio Abra de Minas: nuevos aportes para la caracterización de las ocupaciones Tardío/Incas en las tierras altas del Noroeste argentino. Bulletin de l'Institut Français d'Études Andines, 44(1), 141-149.

" López, G., Coloca, F., Araya, S., Orsi, J. P. y Seguí, S. (2015). El sitio Cueva Inca Viejo, salar de Ratones, Puna de Salta: Evidencia arqueológica y procesos de interacción macro-regional. Relaciones de la Sociedad
Argentina de Antropología, XL(1), 45-71.

» López, G., Coloca, F. y Orsi, J. P. (2009). Ocupaciones humanas holocénicas en abrigos rocosos de la Puna de Salta. Comechingonia, 12, 109-115.

» Martel, A. (2011). El espacio ritual pastoril y caravanero. Una aproximación desde el arte rupestre de valle Encantado (Salta, Argentina). En L. Núñez y A. Nielsen (Eds.), En ruta. Arqueología, historia y etnografía del tráfico sur andino (pp. 111-15o). Córdoba: Encuentro.

»Nielsen, A. E. (2003). La edad de los auca runa en la Quebrada de Humahuaca (Jujuy, Argentina). Memoria Americana, 11, 73-109.

» Pérez Gollán, J. A. (1973). Arqueología de las culturas agroalfareras de la Quebrada de Humahuaca (Provincia de Jujuy, República Argentina). América Indígena, 33(3), 667-679.

»Raffino, R. (1981). Los Inkas del Kollasuyu. La Plata: Ramos Americana.

" Richerson, P. y Boyd, R. (2000). The Evolution of Subjective Commitment to Groups: A Tribal Instincts Hypothesis. En R. Nesse (Ed.), The Evolution of Subjective Commitment, Russell Sage Foundation (pp.1-32). Nueva York: Russell Sage Foundation.

» Richerson, P. y Henrich, J. (2009). Tribal social instincts and the cultural evolution of institutions to solve collective action problems, http://ssrn.com/ abstract=1368756. (Acceso: junio, 2017).

»Salazar, D., Berenguer, J. y Vega, G. (2013). Paisajes minero-metalúrgicos inkaicos en Atacama y el Altiplano sur de Tarapacá (norte de Chile). Chungara. Revista de Antropología Chilena, 45(1), 83-103.

»Soto Rodríguez, C. y Salazar, D. (2016). Mullu (Spondylus sp.) en el Complejo Minero San José del Abra (Alto Loa, Norte de Chile). Intersecciones en Antropología, 17, 129135 .

» Tarragó, M. (2000). Chacras y pukará. Desarrollos sociales tardíos. En Nueva Historia Argentina. Los pueblos originarios y la conquista (Tomo 1) (pp. 257-300). Ciudad Autónoma de Buenos Aires: Editorial Sudamericana.

» Van Kessel, J. (1989). Ritual de producción y discurso tecnológico. Chungara. Revista de Antropología Chilena, 23, 73-91.

»Williams, V., Villegas, M., Gheggi, M. y Chaparro, M. (2005). Hospitalidad e intercambio en los valles mesotermales del Noroeste Argentino. Boletín de Arqueología PUCP, 9, 335-373. 